$\xi=\mathrm{\alpha}$

\title{
Temporal sleep patterns between healthy and depressed individuals
}

\author{
Mohammad A. Alotaibi, Mark Halaki, Chin Moi Chow* \\ Exercise, Health \& Performance Faculty Research Group, Faculty of Health Sciences The University of Sydney, \\ 75 East Street, Lidcombe, NSW 2141 \\ *Corresponding author E-mail: chin-moi.chow@sydney.edu.au
}

\begin{abstract}
Background: Individuals with depression differ in their sleep patterns from healthy subjects. However, there are no studies that compare the temporal sleep patterns between healthy and depressed people or explore the relations between depressive symptomatology and physical activity levels. This study is an attempt to address this gap.

Method: Participants recruited were 20 healthy and 20 depressed individuals. Data related to sleep-wake patterns, and activity levels were collected over four weeks using actigraph device (Actiwatch 2) and depressive symptoms were collected using the Depression Anxiety and Stress Scale (DASS) and Quick Inventory of Depressive Symptoms (QIDS) questionnaire. The data for the two groups were compared using t-tests. Correlation analyses were employed to test for associations between depressive symptoms, activity level and sleep patterns for each group.

Result: The depressed group had significantly higher scores for depression, longer total sleep time, and lower level of activity compared to the healthy group. Sleep onset latency (SOL) was significantly correlated with the anxiety subscale of DASS, the depression score as measured by QIDS, and the activity level in the depressed group. The positive association between activity level and SOL was anomalous, and did not reflect the expected pattern seen in healthy individuals.

Conclusion: The depressed group was confirmed to have significantly higher levels of depression, stress and anxiety, and lower level of physical activity. Increased anxiety and depression predicted delayed sleep onset. Depressive symptoms may have masked the expected relation between physical activity and SOL seen in healthy individuals.
\end{abstract}

Keywords: Actigraphy; Depressive Symptoms; Physical Activity; Sleep Onset Latency.

\section{Introduction}

Sleep is vital to human health. Sleep enhances the physical restoration of the body [1] and re-establishes the mind. Indeed, sleep is an important life-style factor that contributes to the overall health and well being. Pilcher and colleagues [2] reported that sleep quality rather than sleep quantity predicted health and well being in college students. According to Riemann [3] the quality of sleep is more important than the quantity. The satisfaction derived from a good sleep is a crucial element for good health as well as physical performance [4]. Notably, the components of sleep quality and sleep quantity overlap somewhat. Sleep quality could be affected by a number of factors such as the length of time to fall asleep, number of Awakenings at night, sleep on duration, time taken to fall back asleep after Awakenings, feeling of restfulness after sleep, and general satisfaction achieved from the bout of sleep [2]. Sleep quantity refers to the first three components listed for sleep quality [2].

Sleep loss impacts on physical, psychological and metabolic health. It causes fatigue [5], irritation, short temperedness, vulnerabilitybtoistresss [6], sand] increasesrtheeriskeofiweightwgaintandidevelopmentoofediabetes, bcardiovascularsandaimmuneiconditions [7], [8]. [7], [8].

Depression and sleep disturbances are closely related and bidirectional [9]. Depression affects brain functions, including emotions, coordination, senses, and the sleep-wake cycle. Depression may cause sleeplessness or excessive sleepiness and disrupt the sleep-wake rhythms and the biologic clock, which adds to the depression [3]. Thus, a vicious cycle of lack of sleep which leads to depression, which leads to more lack of sleep and so forth may be established in many individuals.

Interruptions in sleep are not desirable. Disturbances in sleep due to depression are characterized by a reduction of slow wave sleep and by a shortening of the phase between sleep onset and the occurrence of the first REM period [3]. The amount of sleep was seen to affect emotional health, although a reduction in sleep quantity was not the only factor that led to depression [10]. However, decreased amount of sleep may implicate decreased quality of sleep. It is thus not surprising that sleep-related disorders, like obstructive sleep apnea and restless leg syndrome, are associated with increased rates of depression [11]. Physical activity may assist in combating both depression [12] and sleep disorders as increased levels of physical activity have been shown to lower the rate of sleep disorders in depressed individuals [13]. Therefore, this study aimed to determine the trends, differences, and to explore associations between the levels of depression, sleep patterns and physical activity levels in both healthy and people with depression.

\section{Methods}

This descriptive study involved collecting and analyzing objective actigraph sleep data over 28 days as well as depression and anxiety questionnaires from 20 healthy and 20 depressed individuals. The study received ethical approval by the Institution Ethics 
Committee (Approval No. 2015/432). Written consent was obtained from all participants.

\subsection{Participants}

The healthy participants ( 5 female and 15 male) had a mean age of $27.4 \pm 7.3$ years and body mass index (BMI) of $23.7 \pm 4.4 \mathrm{~kg} . \mathrm{m}^{-2}$ All twenty participants in the depressed group were males and had a mean age of $29.0 \pm 6.8$ years and a BMI of $25.7 \pm 4.6 \mathrm{~kg} . \mathrm{m}^{-2}$.

\subsection{Procedure}

Healthy participants were recruited by in-class announcements and advertisements posted at the University. Participants with diagnosed major depressive disorder were referred by a psychiatrist not involved in the study. Participants wore asleep and activity motion logger (Actiwatch 2, Philips Respironics, Murrysville, PA, USA) for sleep-wake pattern recording. All participants attended the laboratory on three occasions: at baseline, at two weeks, and at four weeks (study exit). At two weeks (mid-way of the study), participants returned to collect a charged replacement Actiwatch 2. All participants completed the Quick Inventory of Depressive Symptomatology (QIDS) and the Depression Anxiety Stress Scale (DASS-D, DASS-A and DASS-S) during each session.

\subsection{Sleep measurements}

Actiwatch 2 is a simple watch-like device, worn on the nondominant wrist and monitors sleep and activity. It has a memory storage device and movement detector. Rest or activity of the wrist is measured by the accelerometer present in the Actiwatch 2 . It gives an estimated measurement for various sleep parameters including: Total Sleep Time (TST): the amount of sleep obtained in total; Sleep Efficiency (SE): the ratio between the total recording time and the total sleep time; Sleep Onset Latency (SOL): how long it takes to fall asleep; Wake After Sleep Onset Latency (WASO): the total time awake during the sleep period. A button located on the side of the Actiwatch 2 acts as an event marker to mark bedtime and rise time. All subjects were asked to press the button once before sleep i.e., when they had turned off the lights and lain down, and again when they got up. In this way, the rest interval or time in bed, marked by bedtime and rise time, was determined. A proprietary algorithm is applied to the rest interval to yield the estimated sleep parameters. Data were collected using the default settings, with a sensitivity level of the algorithm for detecting wake at medium level and epoch lengths of $30 \mathrm{~s}$ [14]. Actiwatch 2 data were analysed with the Respironics Actiware v5.59.0015 [14]. Average scores were calculated for all the sleep variables (TST, SE, SOL and WASO) for each individual across the data collection period of 28 days to obtain representative figures which could be used for analysis

\subsection{Statistical analysis}

To express the central tendency of the collected data, all values were presented as the mean \pm standard deviation (SD) and $95 \%$ confidence interval. For assessing the variation in measurements between healthy and depressed group, Student's t-tests for independent samples were used. Pearson's correlation analyses were performed to explore associations between different measures of depression, activity count, and sleep pattern for both healthy and depressed groups. Furthermore, the study used a two-step cluster analysis using the Akaike's information criterion (AIC) to establish if the temporal sleep characteristics of TST, SE, SOL and WASO could be clustered into depressed and healthy groups. Three different cluster analyses were conducted. The first included mean TST, SOL and WASO; in the second analysis, WASO was replaced by SE [15] and the third used 28 consecutive records of TST. The silhouette coefficient (range -1 to +1 ) was used to assess the quality of clustering. The data were evaluated using Microsoft Excel and SPSS statistical software version 22. Statistical test results with $\mathrm{p}$-value $<0.05$ were considered significant.

\section{Results}

There was no significant difference between healthy and depressed groups with respect to demographic or anthropometric variables (Table 1). Significant differences were obtained for all the depression variables (QIDS, DASS-D, DASS-A and DASS-S) with the depressed group recording significantly higher values compared to the healthy group (Table 1). Among sleep variables (Table 1), the depressed group had significantly higher TST values compared to healthy group with no other differences in sleep variables. Activity count was significantly lower for the depressed group compared to the healthy group.

Table 2 shows the correlation results for different measures of depression and sleep variables and activity count for both healthy (H) and depressed (D) group. SOL was significantly associated with anxiety (DASS-A), QIDS and activity count in the depressed group. All the other variables were not significantly correlated (Table 2).

Table 3 shows mean individual data for the mean sleep variables over 28 consecutive nights and these data were employed in the cluster analysis.

Table 1: Demographics, Depression and Sleep Characteristics of Healthy and Depressed Subjects

\begin{tabular}{|c|c|c|c|c|c|}
\hline & $\begin{array}{l}\text { Healthy } \\
\mathrm{n}=20 \\
\text { Mean } \pm \text { SD }\end{array}$ & $95 \% \mathrm{CI}$ & $\begin{array}{l}\text { Depressed } \\
\mathrm{n}=20 \\
\text { Mean } \pm \text { SD }\end{array}$ & $95 \% \mathrm{CI}$ & $P$ value \\
\hline Age (year) & $27.4 \pm 7.3$ & $24.0-30.8$ & $29.0 \pm 6.8$ & $25.8-32.2$ & 0.476 \\
\hline \multicolumn{6}{|l|}{ Anthropometric Variables } \\
\hline Height $(\mathrm{cm})$ & $169.6 \pm 7.8$ & $165.9-173.2$ & $171.2 \pm 7.5$ & $167.7-174.7$ & 0.500 \\
\hline Weight (kg) & $68.2 \pm 15.2$ & $61.1-75.3$ & $75.8 \pm 15.9$ & $68.3-83.2$ & 0.135 \\
\hline Waist circumference $(\mathrm{cm})$ & $81.4 \pm 12.3$ & $75.7-87.1$ & $84.9 \pm 19.3$ & $75.8-93.9$ & 0.504 \\
\hline BMI (kg/m2) & $23.7 \pm 4.4$ & $21.6-25.7$ & $25.7 \pm 4.6$ & $23.6-27.9$ & 0.157 \\
\hline \multicolumn{6}{|l|}{ Depression Variables } \\
\hline QIDS & $1.9 \pm 1.9$ & $1.0-2.7$ & $16.4 \pm 2.6$ & $15.1-17.6$ & $<0.001^{*}$ \\
\hline DASS-D & $1.4 \pm 1.8$ & $0.5-2.2$ & $12.5 \pm 2.4$ & $11.4-13.5$ & $<0.001^{*}$ \\
\hline DASS-A & $1.2 \pm 1.5$ & $0.5-1.9$ & $4.9 \pm 3.6$ & $3.2-6.6$ & $<0.001 *$ \\
\hline DASS-S & $1.6 \pm 3.0$ & $0.2-3.0$ & $5.0 \pm 3.2$ & $3.5-6.4$ & $<0.001^{*}$ \\
\hline \multicolumn{6}{|l|}{ Sleep Variables } \\
\hline TST (min) & $393.6 \pm 37.8$ & $375.9-411.3$ & $455.0 \pm 79.1$ & $418.0-492.0$ & $0.004 *$ \\
\hline SE $(\%)$ & $81.5 \pm 5.3$ & $79.1-84.0$ & $82.4 \pm 6.1$ & $79.6-85.3$ & 0.617 \\
\hline SOL (min) & $20.4 \pm 9.6$ & $15.9-25.0$ & $26.2 \pm 27.9$ & $13.1-39.2$ & 0.394 \\
\hline WASO (min) & $48.0 \pm 21.1$ & $38.1-57.9$ & $44.3 \pm 19.7$ & $35.1-53.5$ & 0.569 \\
\hline Activity Count (units) & $\begin{array}{l}306,139 \pm \\
72,583\end{array}$ & $\begin{array}{l}272,169- \\
340,109\end{array}$ & $257,937 \pm 55,864$ & $\begin{array}{l}231,792- \\
284,082\end{array}$ & $0.024 *$ \\
\hline
\end{tabular}


TST: Total Sleep Time; SE: Sleep Efficiency; SOL: Sleep Onset Latency; WASO: Wake After Sleep Onset; QIDS: Quick Inventory of Depressive Symptomatology; DASS-D: Depression Anxiety and Stress Scale - Depression; DASS-A: Depression Anxiety and Stress Scale - Anxiety; DASS-S: Depression Anxiety and Stress Scale - Stress

* And bold indicates significant differences

Table 2: Pearson's Correlation Coefficient (R) Between Level of Depression, Sleep Pattern and Activity Count

\begin{tabular}{|c|c|c|c|c|c|c|c|c|c|c|c|}
\hline \multirow{3}{*}{ Variable } & & \multicolumn{8}{|c|}{ Measurement of Depression } & \multirow{2}{*}{\multicolumn{2}{|c|}{ Activity Count }} \\
\hline & & \multicolumn{2}{|c|}{ DASS-D } & \multicolumn{2}{|c|}{ DASS-A } & \multicolumn{2}{|c|}{ DASS-S } & \multicolumn{2}{|l|}{ QIDS } & & \\
\hline & & $\mathrm{H}$ & $\mathrm{D}$ & $\mathrm{H}$ & $\mathrm{D}$ & $\mathrm{H}$ & $\mathrm{D}$ & $\mathrm{H}$ & $\mathrm{D}$ & $\mathrm{H}$ & $\mathrm{D}$ \\
\hline $\begin{array}{l}\text { TST } \\
\text { (min) }\end{array}$ & r value & 0.39 & 0.13 & 0.18 & 0.18 & 0.35 & 0.24 & 0.01 & 0.06 & -0.34 & 0.40 \\
\hline $\mathrm{SE}(\%)$ & $\begin{array}{l}\mathrm{p} \text { value } \\
\mathrm{r} \text { value } \\
\mathrm{p} \text { value }\end{array}$ & $\begin{array}{l}0.09 \\
0.07 \\
0.76\end{array}$ & $\begin{array}{l}0.57 \\
0.28 \\
0.22\end{array}$ & $\begin{array}{l}0.44 \\
0.01 \\
0.98\end{array}$ & $\begin{array}{l}0.45 \\
0.08 \\
0.73\end{array}$ & $\begin{array}{l}0.13 \\
0.04 \\
0.86\end{array}$ & $\begin{array}{l}0.31 \\
0.22 \\
0.34\end{array}$ & $\begin{array}{l}0.95 \\
0.41 \\
0.08\end{array}$ & $\begin{array}{l}0.81 \\
0.05 \\
0.85\end{array}$ & $\begin{array}{l}0.14 \\
-0.15 \\
0.52\end{array}$ & $\begin{array}{l}0.08 \\
0.25 \\
0.29\end{array}$ \\
\hline $\begin{array}{l}\text { SOL } \\
(\min )\end{array}$ & r value & 0.02 & 0.11 & 0.09 & $0.54^{*}$ & 0.25 & 0.41 & 0.03 & $0.54 *$ & -0.01 & $0.50 *$ \\
\hline & $\mathrm{p}$ value & 0.95 & 0.64 & 0.69 & $0.01 *$ & 0.29 & 0.07 & 0.90 & $0.01 *$ & 0.97 & $0.03 *$ \\
\hline $\begin{array}{l}\text { WASO } \\
(\mathrm{min})\end{array}$ & r value & 0.31 & 0.33 & -0.16 & -0.18 & -0.31 & 0.00 & -0.37 & -0.04 & 0.08 & -0.17 \\
\hline Activity Count & $\begin{array}{l}\mathrm{p} \text { value } \\
\text { r value } \\
\mathrm{p} \text { value }\end{array}$ & $\begin{array}{l}0.18 \\
-0.02 \\
0.94\end{array}$ & $\begin{array}{l}0.16 \\
-0.05 \\
0.83\end{array}$ & $\begin{array}{l}0.50 \\
0.10 \\
0.68\end{array}$ & $\begin{array}{l}0.44 \\
0.29 \\
0.21\end{array}$ & $\begin{array}{l}0.19 \\
0.16 \\
0.50\end{array}$ & $\begin{array}{l}0.99 \\
0.07 \\
0.76\end{array}$ & $\begin{array}{l}0.11 \\
0.02 \\
0.92\end{array}$ & $\begin{array}{l}0.86 \\
-0.19 \\
0.42\end{array}$ & 0.75 & 0.47 \\
\hline
\end{tabular}

*And bold indicate significant rvalues; H: Healthy, D: Depressed

Table 3: Data for TST, SE, SOL and WASO Averaged Over 28 Consecutive Days

\begin{tabular}{|c|c|c|c|c|}
\hline Subjects & TST & SE (\%) & SOL (min) & WASO (min) \\
\hline$\overline{\mathrm{H}-1}$ & 337.4 & 84.0 & 6.5 & 37.1 \\
\hline $\mathrm{H}-2$ & 453.3 & 89.1 & 14.6 & 36.3 \\
\hline $\mathrm{H}-3$ & 446.7 & 84.3 & 5.8 & 35.4 \\
\hline $\mathrm{H}-4$ & 425.7 & 81.8 & 15.8 & 45.8 \\
\hline $\mathrm{H}-5$ & 428.7 & 88.3 & 15.8 & 35.0 \\
\hline $\mathrm{H}-6$ & 389.4 & 80.0 & 26.0 & 57.5 \\
\hline $\mathrm{H}-7$ & 379.4 & 82.8 & 15.8 & 30.5 \\
\hline H-8 & 405.0 & 89.8 & 22.0 & 22.4 \\
\hline H-9 & 393.3 & 90.3 & 14.4 & 25.1 \\
\hline $\mathrm{H}-10$ & 371.4 & 86.4 & 19.7 & 19.8 \\
\hline $\mathrm{H}-11$ & 346.0 & 76.7 & 36.0 & 38.6 \\
\hline $\mathrm{H}-12$ & 311.6 & 74.7 & 25.0 & 47.0 \\
\hline H-13 & 364.8 & 75.5 & 13.5 & 69.0 \\
\hline $\mathrm{H}-14$ & 419.9 & 76.4 & 19.3 & 46.3 \\
\hline H-15 & 421.0 & 81.3 & 28.3 & 41.0 \\
\hline H-16 & 397.1 & 78.5 & 27.8 & 64.0 \\
\hline $\mathrm{H}-17$ & 407.0 & 83.1 & 20.5 & 51.6 \\
\hline H-18 & 349.8 & 75.7 & 14.8 & 79.6 \\
\hline H-19 & 426.0 & 75.4 & 47.3 & 100.3 \\
\hline $\mathrm{H}-20$ & 398.9 & 76.8 & 20.2 & 77.9 \\
\hline D-1 & 356.2 & 80.7 & 26.0 & 61.3 \\
\hline D-2 & 393.5 & 81.9 & 27.6 & 37.9 \\
\hline D-3 & 521.6 & 91.7 & 4.5 & 8.6 \\
\hline D-4 & 580.1 & 87.1 & 120.8 & 57.8 \\
\hline D-5 & 477.1 & 82.7 & 68.6 & 44.8 \\
\hline D-6 & 420.0 & 75.5 & 31.9 & 39.3 \\
\hline D-7 & 474.0 & 83.1 & 50.8 & 38.3 \\
\hline D-8 & 424.2 & 84.3 & 31.3 & 40.0 \\
\hline D-9 & 419.9 & 84.5 & 20.7 & 34.3 \\
\hline D-10 & 466.7 & 77.8 & 39.0 & 59.0 \\
\hline D-11 & 509.4 & 91.1 & 14.1 & 11.8 \\
\hline D-12 & 372.6 & 78.1 & 14.0 & 66.4 \\
\hline D-13 & 416.2 & 80.1 & 12.8 & 52.4 \\
\hline D-14 & 302.0 & 68.3 & 13.9 & 56.5 \\
\hline D-15 & 470.1 & 80.5 & 12.8 & 75.5 \\
\hline D-16 & 613.8 & 95.0 & 2.8 & 16.7 \\
\hline D-17 & 443.1 & 76.0 & 10.3 & 68.7 \\
\hline D-18 & 375.3 & 85.2 & 7.8 & 33.1 \\
\hline D-19 & 567.0 & 80.2 & 4.0 & 20.0 \\
\hline D-20 & 496.9 & 85.1 & 9.8 & 63.8 \\
\hline
\end{tabular}

The cluster analysis identified 2 clusters in all three cases. For TST, SOL, WASO: 10 depressed subjects (D3,4,5,7,10,11,15,16,19,20) and 1 healthy subject (H19) formed a cluster while the rest of the depressed and the healthy subjects formed another cluster (Fig.1). The Silhouette measure of cohesions and separation $=0.4$ (Fair) and AIC was 82.1.

- Cluster $1 \circ$ Cluster2 


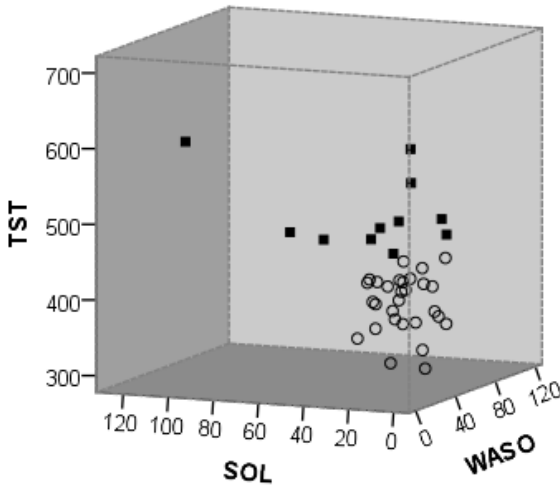

Fig. 1: Clustering Using TST, SOL and WASO.

For TST, SOL, SE: 8 subjects (a subset of the 10 depressed people from Cluster 1 in the previously described cluster analysis: 8 depressed $(\mathrm{D} 3,4,5,7,10,11,15,16,19,20)$ formed the first cluster while the rest of the depressed and all the healthy formed another cluster (Fig.2). The average Silhouette measure of cohesions and separation $=0.5(\mathrm{Good})$ and AIC was 82.1.

\section{Cluster $1 \bigcirc$ Cluster 2}

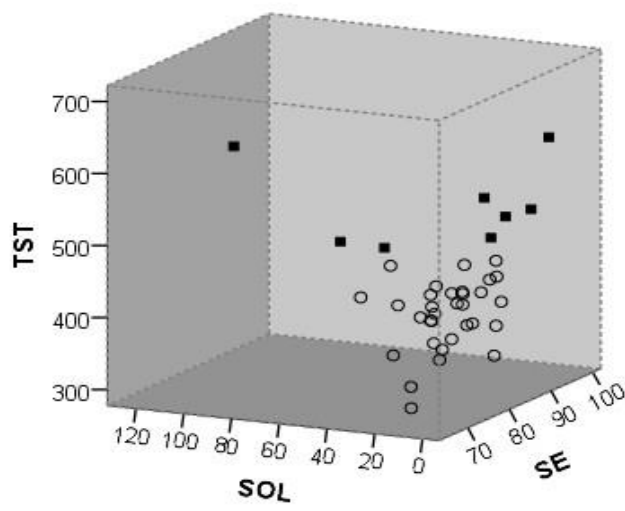

Fig. 1: Clustering using TST, SOL and SE.

For TST over 28 days: 6 subjects (a subset of the 10 depressed people from the Cluster 1 in the previously described cluster analysis: (D3, 4, 5, 7, 10, 11, 15, 16, 19, 20) formed a cluster while the rest of the depressed and the healthy subjects formed another cluster. The average Silhouette measure of cohesions and separation = 0.4 (Fair) and the AIC was11974.2.

\section{Discussion}

\subsection{Confirming depression in the depressed group}

We confirm that participants in the depressed group showed high scores for all DASS subscales of depression, anxiety and stress as well as the QIDS, which classified them as clinically depressed (see Table 1). Their scores were statistically significantly different from the healthy group. These data establish baseline differences in the mental health of the two groups. These finding suggest that any subsequent differences between the groups could be due to the mental health differences.

\subsection{Sleep parameters}

It is interesting to note that, in the current study, the only sleep parameter that was significantly different between the depressed and healthy groups was TST which was lower for the healthy group. These findings, although contrary to the findings of some similar research described below, are similar to others. Depression was linked to lower TST, sleep efficiency [10], [16] and longer
SOL [10], [17] and WASO [10] as well as light and fragmented sleep and early morning awakening [18]. However, depression has also been reported to cause oversleeping [19], [20]. In this research, the depressed group on average slept for approximately 7.5 hours compared to the healthy group who slept for approximately 6.5 hours. It is noteworthy that 7.5 hours may be considered long in this group of adults (mean age of $29.0 \pm 6.8$ years), since people in this age group often incurred a partial sleep loss [21].

Although non-significant differences in SOL were observed between the healthy and depressed groups, a delayed sleep onset predicted an increased depression in the depressed group (Table 2). Furthermore, delayed sleep onset also predicted increased anxiety (as measured by the subscale of DASS-A) and depression (by QIDS). Our finding is consistent with previous studies: impaired sleep (of which SOL is a dimension) was shown to be associated with depression [3], [22-25] and anxiety [23]. According to Mansfield [26], stress and anxiety may cause issues with sleep or make existing sleep problems worse. Indeed, sleep problems (e.g. SOL) may also be the first sign of an imminent episode of major depression [27].

\subsection{Physical activity levels}

In the current study, depressed people had significantly lower activity levels compared to healthy people. Several reports indicated that increased physical activity predicted less depression [13], [28], [29] and vice versa [30-32]. We found a significant positive association between activity levels and SOL for depressed people. This result appears counterintuitive as one would expect a greater ease of falling asleep with increased level of activity [33]. Furthermore, exercise duration did not have an effect on SOL as reported in a meta-analysis study acute exercise on sleep [34]. However, the depressed group incurred lower level of physical activity than the healthy group. They also had a high level of anxiety and depression, which significantly delayed sleep onset. Thus, depressive symptoms may have masked the expected relation between physical activity and SOL seen in healthy individuals.

It should be noted that the depressed group had a larger level of dispersion (as measured by the standard deviation) for SOL compared to the healthy group. It is possible that there may be other extraneous variables responsible for this finding, which has not been considered throughout this study.

\subsection{Cluster analysis}

The cluster analysis was successful in clustering the sample into two clusters based on the mean TST, WASO and SOL; mean TST, SE and SOL; or TST over the 28 days alone. The cluster analysis showed that almost half of the depressed subjects had similar associations among the sleep parameters as well as similar TST over 28 days as healthy controls. Although cluster analysis on sleep variables (TST, SE, WASO and SOL) could be used to identify people with depression, the accuracy of correctly clustering around the two groups were, on an average, less than $45 \%$ in the three instances. This indicates two things: sleep on variables of TST, SE, SOL and WASO have the potential to predict depression and there may be other sleep variables not considered within this study, which could improve the predictive accuracy of the clustering, for example, slow wave sleep and REM sleep derived from polysomnography. Future research can take into consideration additional sleep variables not used in this study. In addition, an increased sample size will improve the predictive accuracy of the clustering.

\section{Conclusion}

This study found some expected and some counter-intuitive results The depressed group was confirmed to have significantly higher levels of depression, stress and anxiety; they were also found to have lower levels of activity. However, it was found that de- 
pressed people slept more (longer TST) compared to healthy people, which is not uncommon. Increased anxiety and depression, and increased activity levels predicted delayed sleep onset of the depressed group which may be a unique characteristic of depression. The cluster analysis, by employing the sleep variables of mean TST, SOL and WASO, has the highest potential to predict depression compared to mean TST, SOL and SE or TST over 28 days.

\section{Acknowledgement}

M. Alotaibi was awarded a PhD scholarship sponsored by SaudiArabian Culture Mission (SACM), Canberra, Australia, who represents the Ministry of Higher Education of the Saudi-Arabian Government.

\section{References}

[1] Edéll-Gustafsson, U., Sleep, psychological symptoms and quality of life in patients undergoing coronary artery bypass grafting. Nordic Journal of Psychiatry, 1999. 53(2): p. 159-162. https://doi.org/10.1080/080394899426882.

[2] Pilcher, J.J., D.R. Ginter, and B. Sadowsky, Sleep quality versus sleep quantity: relationships between sleep and measures of health, well-being and sleepiness in college students. Journal of psychosomatic research, $1997 . \quad 42(6):$ p. 583-596. https://doi.org/10.1016/S0022-3999(97)00004-4.

[3] Riemann, D., Berger M, and U. Voderholzer, Sleep and depression a results from psychobiological studies: an overview. Biol Psychol 2001. 57((1-3)): p. 76-103. https://doi.org/10.1016/S03010511(01)00090-4

[4] Heslop, P., et al., Sleep duration and mortality: the effect of short or long sleep duration on cardiovascular and all-cause mortality in working men and women. Sleep medicine, 2002. 3(4): p. 305-314. https://doi.org/10.1016/S1389-9457(02)00016-3.

[5] Owens, J.A., Sleep loss and fatigue in healthcare professionals. The Journal of perinatal \& neonatal nursing, 2007. 21(2): p. 92-100. https://doi.org/10.1097/01.JPN.0000270624.64584.9d.

[6] Benca, R.M., et al., Sleep and mood disorders. Sleep medicine reviews, 1997. 1(1): p. 45-56. https://doi.org/10.1016/S10870792(97)90005-8.

[7] Levy, P., et al., Sleep deprivation, sleep apnea and cardiovascular diseases. Frontiers in bioscience (Elite edition), 2011. 4: p. 2007 2021.

[8] Patel, S.R. and F.B. Hu, Short sleep duration and weight gain: a systematic review. Obesity, 2008. 16(3): p. 643-653. https://doi.org/10.1038/oby.2007.118.

[9] Jansson-Fröjmark, M. and K. Lindblom, A bidirectional relationship between anxiety and depression, and insomnia? A prospective study in the general population. Journal of psychosomatic research, 2008. 64(4): p. 443-449. https://doi.org/10.1016/j.jpsychores.2007.10.016.

[10] Gupta, R., S. Dahiya, and M.S. Bhatia, Effect of depression on sleep: Qualitative or quantitative? Indian journal of psychiatry, 2009. 51(2): p. 117-121. https://doi.org/10.4103/0019-5545.49451.

[11] Gupta, M.A., F.C. Simpson, and D.C. Lyons, The effect of treating obstructive sleep apnea with positive airway pressure on depression and other subjective symptoms: A systematic review and metaanalysis. Sleep medicine reviews, 2016. 28: p. 55-68. https://doi.org/10.1016/j.smrv.2015.07.002

[12] Lang, C., et al., The relationship between physical activity and sleep from mid adolescence to early adulthood. A systematic review of methodological approaches and meta-analysis. Sleep medicine $\begin{array}{llll}\text { reviews, } 2016 . & 28: & \text { p. } & 32-45 .\end{array}$ https://doi.org/10.1016/j.smrv.2015.07.004.

[13] Baumgartner, A. and N. Sucher, The influence of physical activity and posture on the antidepressant effect of sleep deprivation in depressed patients. Journal of affective disorders, 1990. 20(2): p. $93-$ 99. https://doi.org/10.1016/0165-0327(90)90122-O.

[14] Respironics, Actiwatch Communication and Sleep Analysis Software with ActiReader: Actiware and Actiware CT Software and Hardware Manual. Murrysville, ed. P. Respironics. 2009, Murrysville, PA; 2009.

[15] Matuzaki, L., et al., Temporal sleep patterns in adults using actigraph. Sleep Science, 2014. 7(3): p. 152-157. https://doi.org/10.1016/j.slsci.2014.09.012.
[16] Irwin, M., T.L. Smith, and J.C. Gillin, Electroencephalographic sleep and natural killer activity in depressed patients and control subjects. Psychosomatic Medicine, 1992. 54(1): p. 10-21. https://doi.org/10.1097/00006842-199201000-00002.

[17] Robillard, R., et al., Delayed sleep phase in young people with unipolar or bipolar affective disorders. Journal of affective disorders, 2013. 145(2): p. 260-263. https://doi.org/10.1016/j.jad.2012.06.006.

[18] Borbély, A. and A. Wirz-Justice, Sleep, sleep deprivation and depression. Hum Neurobiol, 1982. 1(205): p. 10.

[19] Giedke, H. and F. Schwarzler, Therapeutic use of sleep deprivation in depression. Sleep Medicine Reviews, 2002. 6(5): p. 361-77. https://doi.org/10.1016/S1087-0792(02)90235-2.

[20] Vogel, G.W., et al., Improvement of depression by REM sleep deprivation: new findings and a theory. Archives of General Psychiatry, $1980 . \quad 37(3)$ : $\quad$ p. $247-253$. https://doi.org/10.1001/archpsyc.1980.01780160017001.

[21] Bonnet, M.H. and D.L. Arand, We are chronically sleep deprived. SLEEP-NEW YORK-, 1995. 18: p. 908-911.

[22] Roane, B.M., et al., The link between sleep disturbance and depression among Mexican Americans: a Project FRONTIER study. Journal of clinical sleep medicine: JCSM: official publication of the American Academy of Sleep Medicine, 2014. 10(4): p. 427-431.

[23] Gunnarsdóttir, K., Effects of Poor Subjective Sleep Quality on Symptoms of Depression and Anxiety among Adolescents. 2014.

[24] Sivertsen, B., et al., The bidirectional association between depression and insomnia: the HUNT study. Psychosomatic medicine, 2012. 74(7): $\quad$ p. https://doi.org/10.1097/PSY.0b013e3182648619.

[25] Roberts, N.P., et al., Multiple session early psychological interventions for the prevention of post-traumatic stress disorder. Cochrane Database of Systematic Reviews, 2010(4): p. 1-43.

[26] Mansfield, R., Sleep as a Remedy for Stress: the Sleep Response at a Cellular Level. 2015, California State University, Northridge.

[27] Murphy, M.J. and M.J. Peterson, Sleep disturbances in depression. Sleep medicine clinics, 2015. 10(1): p. 17-23. https://doi.org/10.1016/j.jsmc.2014.11.009.

[28] Reid, K.J., et al., Aerobic exercise improves self-reported sleep and quality of life in older adults with insomnia. Sleep medicine, 2010. 11(9): p. 934-940. https://doi.org/10.1016/j.sleep.2010.04.014.

[29] Strawbridge, W.J., et al., Physical activity reduces the risk of subsequent depression for older adults. American journal of epidemiology, 2002. 156(4): p. 328-334. https://doi.org/10.1093/aje/kwf047.

[30] Li, Y.-N., et al., Association between quality of life and anxiety, depression, physical activity and physical performance in maintenance hemodialysis patients. Chronic Diseases and Translational $\begin{array}{llll}\text { Medicine, } & 2016 . & 2(2): & \text { p. }\end{array}$ https://doi.org/10.1016/j.cdtm.2016.09.004.

[31] McDowell, C.P., C. MacDonncha, and M.P. Herring, Brief report: Associations of physical activity with anxiety and depression symptoms and status among adolescents. Journal of Adolescence, 2017. 55: p. 1-4. https://doi.org/10.1016/j.adolescence.2016.12.004.

[32] McMahon, E.M., et al., Physical activity in European adolescents and associations with anxiety, depression and well-being. European child \& adolescent psychiatry, 2017. 26(1): p. 111-122. https://doi.org/10.1007/s00787-016-0875-9.

[33] Youngstedt, S.D. and J.D. Freelove-Charton, Exercise and sleep. Exercise, Health, and Mental Health: Emerging Relationships. New York, NY: Routledge, 2005: p. 159-189.

[34] Youngstedt, S.D., P.J. O'Connor, and R.K. Dishman, from wake to sleep. The effects of acute exercise on sleep: a quantitative synthesis. Sleep, 1997. 20(3): p. 203-214. 